\title{
A New Lifetime Prediction Method Based on Particulate Contaminants for Coaxial Connectors
}

\author{
Yuqi Zhou, Yanshen Su,Qingya Li, Qiuyan Jin and Jinchun Gao \\ Beijing University of Posts and Telecommunications, 10 Xitucheng Rd, Beijing 100876, China
}

\begin{abstract}
The high concentration of dust particles in the environment is one of the main causes resulting for electrical contact failure of coaxial connectors. Therefore, it is important to carry out proper methods to predict life time of coaxial connectors. In the life time investigation of coaxial connectors, a model is designed and analyzed. Then conclusions are drawn based on a novel accelerated life test. In this paper, a life time prediction method is proposed based on an $1 \mu \mathrm{m}$-diameter particle environment for the first time, and at the same time a kind of experiment operating equipment is designed to control the concentration of particles for accelerated testing. The result shows an exponential change in contact resistance of coaxial connectors under $1 \mu \mathrm{m}$-diameter particle environment. We then establish Inverse Power Law-Weibull model for life time prediction in electrical contact failure and obtain the contact reliability and characteristic life.
\end{abstract}

Keywords-coaxial connectors; lifetime prediction; inverse power law-weibull model; contact resistance

\section{INTRODUCTION}

Severe air pollution will result in electric performance degradation and reduced reliability of coaxial connectors, especially contaminants like dust and corrosive gases [1][2]. This reduced reliability of coaxial connectors which are the elementary components, may then exacerbate the reliability of the whole communication system. Previous studies [3] show that electric contact failure is one of the main reasons resulting in Bit Error Rate (BER) of communications, computer networks and control systems in China. Therefore, it is theoretically and practically important to make effective life time predictions of electrical contact failure in the fields of connector design and engineering applications.

The research of electrical degradation of coaxial connectors essentially established models based on the relationships between parameters indicating degradation and those indicating electrical reliability [4]. Among different kinds of research, some researchers adapt Weibull distribution and Maximum Likelihood Estimation (ML) to solve the value of degradation parameters [5]. The electrical connector research in China started late and had a large gap between some developed countries, especially in those accelerated life tests in single stress [6] or temperature-humidity-combined stresses' environment [7]which were unparalleled to the real world environment. However, these tests didn't take into consideration degradation in long-time environment, and no accelerated life test in the stress of dust particles.

In this experiment we propose a method based on $1 \mu \mathrm{m}$ diameter contaminants for the first time, and design a kind of experiment operating equipment to control the concentration of particles for accelerated testing. Based on this equipment, we carry out a series of accelerated life tests to obtain the changing curves of degradation parameters in contaminated environment.

\section{EXPERIMENT}

\section{A. Descriptions of Test Samples}

In the experiment we use coaxial connectors, which are widely used in microwave devices and RF circuits, as test samples. The samples are divided into two parts, the coaxial pins (male connectors) and coaxial receptacles (female connectors). Two pins can be inserted snugly into two sides of a receptacle. The combination of pins and receptacles are used in the transmission of high-frequency signal and $\mathrm{AC} / \mathrm{DC}$ systems. The reduced pressure and degradation of the contact spots of pins and receptacles mainly result in the increase of contact resistance, which we adopt four-terminal sensing to measure.

We choose mineral silicon $\left(\mathrm{SiO}_{2}\right)$ particles with a diameter of $1 \mu \mathrm{m}$ as contaminants because our previous experiment showed that particles with these size have a significant impact on the electric performance of connectors. The concentration of particles is set as the stress level in the experiment. Since dry $\mathrm{SiO}_{2}$ does not have a stable attachment to the surface of connectors, $\mathrm{SiO}_{2}$ normally combines with salty fog when stably attached to the surface in the real-world degradation of connectors.

\section{B. Experimental Equipment}

We make an experiment operating box (see Figure 1) to control the concentration of particles more precisely. The box has a volume of $824.03 \mathrm{~cm}^{3}$, with the length of $12.41 \mathrm{~cm}$, the width of $7.58 \mathrm{~cm}$ and the height of $8.76 \mathrm{~cm}$. There are two electrical fans on one side of the box, and on the other side four holes in which coaxial connectors are set. The top of the box has a hole to add particles. During the experimental procedure, we change the concentration level by adding different mass levels of $1 \mu \mathrm{m}$-diameter particles. 


\section{Experimental Procedure}

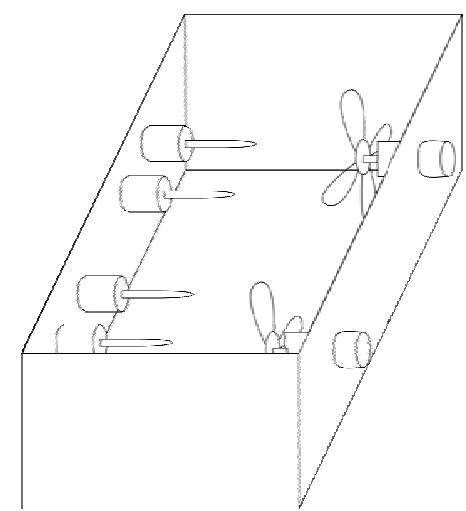

FIGURE I EXPERIMENTAL EQUIPMENT, AN OPERATING BOX

We divide coaxial connector samples into 3 groups, the concentrations of $\mathrm{SiO}_{2}$ particles of which are $1.21 \mathrm{mg} / \mathrm{cm}^{3}$, $1.82 \mathrm{mg} / \mathrm{cm}^{3}$ and $2.42 \mathrm{mg} / \mathrm{cm}^{3}$ respectively. (That means the $\mathrm{SiO}_{2}$ mass of 3 groups is $1.0 \mathrm{~g}, 1.5 \mathrm{~g}, 2.0 \mathrm{~g}$ respectively.) Before the experiment, each group has 1 testing pin, 4 coaxial pin samples and 4 receptacle samples. Each of pin and receptacle samples is numbered from 1 to 4 , respectively. During the experiment, electrical fans are powered on and blow the particles evenly into the air for 2 minutes. In each time cycle, 4 pins are put into the box for corrosion for 1 minute. Then we take the 4 pins out of the box, insert them into 4 receptacles and measure their contact resistance using fourterminal sensing method. We obtain the average contact resistance of 6 times for each pin. After that, 4 pins are put back into the box to be corroded for 1 minute. We continue the procedure above until the $8^{\text {th }}$ minute.

\section{Testing Method}

In this paper, four-terminal sensing is used to measure the contact resistance of each sample, as shown in Figure 2. This method uses a constant current source, measuring the voltage between two contact spot and calculating the contact resistance with Ohm's Law.

\section{LIFETIME PREDICTION}

Generally, in order to predict the lifetime characteristics of electrical connectors in normal environment, high stress conditions should be set in the experiment. Therefore, we establish the relationship between the characteristic life and the concentration stress.

We adopt Inverse Power Law model as accelerated test model. In the lifetime prediction, since the life of electrical connectors obey Weilbull distribution, their life in accelerated stress condition also obey Weilbull distribution. Therefore, we name this lifetime prediction model the "Inverse Power LawWeibull model".

\section{A. Inverse Power Law Model}

The mathematical form of Inverse Power Law model is:

$$
\varepsilon=A S^{-\beta}
$$

where $A$ is a constant, $\beta$ is a constant, $S$ is the stress of particle concentration and $\varepsilon$ stands for the characteristic life.

Solve the exponential equation above in terms of natural logarithms:

$$
\ln \varepsilon=a_{0}+a_{1} \varphi_{1}(S)
$$

where $a_{0}=\ln A, a_{1}=-\beta, \varphi_{1}(s)=\ln S$.

\section{B. Weibull Distribution}

This paper chooses 2-parameter Weibull distribution as the life distribution of coaxial connectors. The failure density function is:

$$
f(t)=\left(\frac{m}{\eta^{m}}\right) t^{m-1} \exp \left[-\left(\frac{t}{\eta}\right)^{m}\right] t, m, \eta>0
$$

\section{Cortact Spot 1}

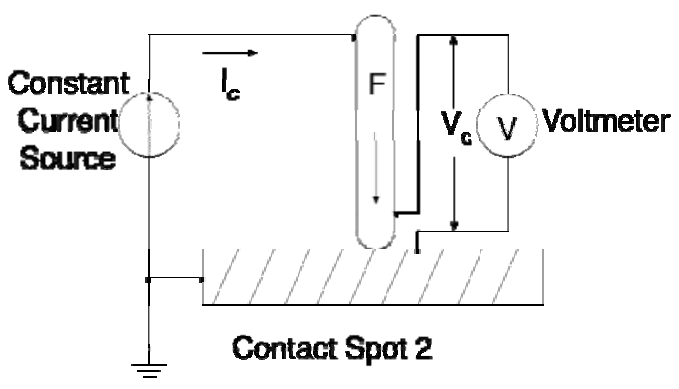

FIGURE II FOUR-TERMINAL SENSING METHOD

where $m$ is a stress-unrelated constant, and $\eta$ is characteristic life.

Cumulative failure function is:

$$
F(\mathrm{t})=1-\exp \left[-\left(\frac{t}{\eta}\right)^{m}\right] t, m, \eta>0
$$

The relationship between characteristic life and concentration stress is:

$$
\ln \eta=a_{0}+a_{1} \varphi_{1}(S)
$$

Putting (5) into (4):

$$
F(t)=1-\exp \left[-\frac{t^{m(S)}}{\exp \left(m(S) A S^{-\beta}\right)}\right]
$$

Change (6) to:

$$
F(t)=1-\exp \left[-\left(\frac{t}{A S^{-\beta}}\right)^{m}\right]
$$


Solve (7) twice in terms of natural logarithms, and the cumulative failure function is:

$$
\ln \ln \frac{1}{1-F(t)}=m(\ln t-\ln A+\beta \ln S)
$$

\section{EXPERIMENTAL RESULTS AND DISCUSSIONS}

\section{A. Experimental Data}

In each concentration level condition (totally 3 concentration levels), the contact resistance of 4 connector samples is measured for every 1 minute (totally 8 minutes). We calculate the average resistance of 6-times measurement for each sample for each minute. The data are shown in Figure 3 , Figure 4 and Figure 5. These figures below show that when the particle concentration is a constant, the contact resistance of coaxial connectors is increased steadily over time. This paper adapts the exponential function $f(t)=a \exp (b x)$ to fit curves on data. The fitting curves are in good agreement with experimental data.

\section{B. Parameter Calculation}

Provided that there are $\mathrm{n}$ samples now, among which there are isamples fail at $t_{i}$ moment. According to IEC255-0-2, the contact reliability is estimated to:

$$
R(t)=1-F(t)=1-\frac{i-0.3}{n+0.4}
$$

Based on the experiment, we consider $R=0.368$ in the following calculation.

The failure threshold resistance of electrical connectors is $20 \mathrm{~m} \Omega$. We fit curves with the data above in exponential function $f(t)=a \exp (b x)$, and obtain the failure time. Putting this failure time and failure threshold resistance into (8), we solve the parameters $m=1.1707, \ln (A)=1.1085, \beta=0.4053$.

\section{Characteristic Life}

According to parameters in Section IV.B, we solve the characteristic life when $R=0.368$.

The concentration of $\mathrm{SiO}_{2}$ is required to be limited to $0.5 \sim 4 \mathrm{mg} / \mathrm{m}^{3}$ in most workplaces. By putting those parameters into (8), we obtain the life function of coaxial connectors:

$$
t=\exp \left[\frac{\ln (-\ln R)}{m}+\ln A-\beta \ln S\right]
$$

When the particle concentration is $0.5 \mathrm{mg} / \mathrm{m} 3$, characteristic life is 12.349day. And when the particle concentration is $4 \mathrm{mg} / \mathrm{m} 3$, characteristic life is 5.3162 day. Therefore, the range of characteristic life is 5.3162 12.3490day.

\section{CONCLUSIONS}

This paper makes an experimental operating box and establishes Inverse Power Law-Weibull model for life time prediction in electrical contact failure. The characteristic life of coaxial connectors is 5.3162 12.3490day, according to experimental data.

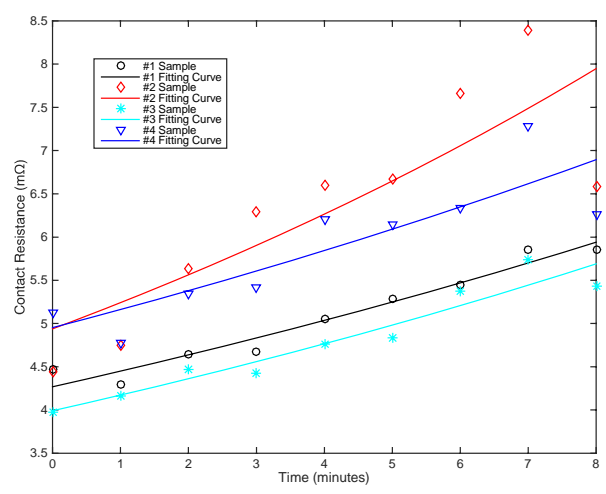

FIGURE III RESISTANCE CHANGE OVER TIME IN PARTICLE CONCENTRATION OF $1.21 \mathrm{mg} / \mathrm{cm} 3$

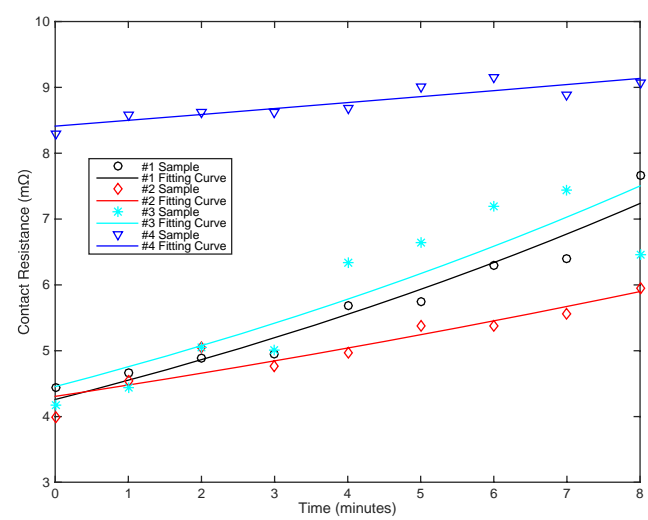

FIGURE IV RESISTANCE CHANGE OVER TIME IN PARTICLE CONCENTRATION OF $1.82 \mathrm{mg} / \mathrm{cm} 3$

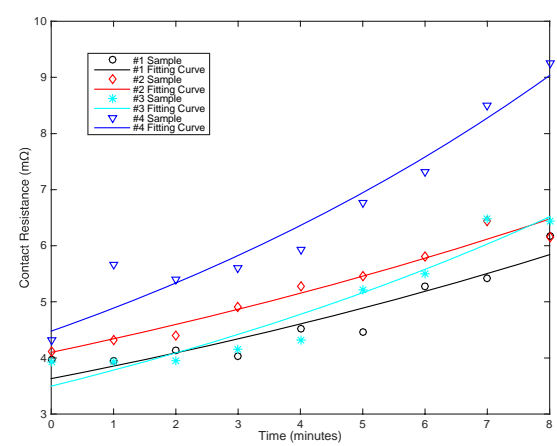

FIGURE V RESISTANCE CHANGE OVER TIME IN PARTICLE CONCENTRATION OF $2.42 \mathrm{mg} / \mathrm{cm} 3$

This paper only studies one stress, the concentration of particles in the air. We will make further investigations studying multi-stress conditions which may include the 
particle size and temperature, and obtain the lifetime prediction methods of coaxial connectors in these conditions.

\section{ACKNOWLEDGMENT}

Beijing University of Posts and Telecommunications student innovation fund sponsors this paper.

\section{REFERENCES}

[1] P. G. Slade, Electrical Contacts Principles and Applications, $2^{\text {nd }} e d$. CRC Press, 2014.

[2] J. G. Zhang, "The effect of environment on electric contact reliability a summary report on recent achievement," Proceedings ICEC, pp. 205211,2002 .

[3] J. G. Zhang, "Effect of dust contamination on electrical contact failure," The 53rd IEEE Holm Conference on Electrical contacts, pp. xxi-xxx, 2007.

[4] T. Y. Wang, Z. G. Li, S. Zuo, Y. Y. Guo and K. Deng, "Degradation modeling and residual life prediction of electronic devices based on Wiener process," ICREPEC, pp. 378-382, 2014.

[5] W. Huang and D. L. Dietrich, "An alternative degradation reliability mode-ling approach maximum likelihood estimation," IEEE Transactions on Reliability, vol. 54(2), pp. 310-317, 2005.

[6] H. W. Wang, T. X. Xu andB. Wang, "Lifetime prediction of missile electrical connector based on Wiener model," Tactical Missile Technology, vol. 35(1), pp. 0042-0045, 2014.

[7] J. Pan, H. J. Liu, W. H. Chen, P. Qian, and J. Liu, "Contact reliability assessment of aerospace electrical connector based on step-up-stress accelerated degradation testing," China Mechanical Engineering, vol. 39(10), pp. 1197-1200, 2011.

[8] X. Y. Huang, "Research on storage accelerated test and life prediction for aerospace relay," Dissertation for the Degree of Master in Engineering, Harbin Institute of Technology, 2013, unpublished 